\title{
Work relations for a system governed by Tsallis statistics
}

\author{
Ian J. Ford and Robert W. Eyre \\ Department of Physics and Astronomy, University College London, Gower Street, London WC1E 6BT, United Kingdom
}

(Received 16 May 2015; published 26 August 2015)

\begin{abstract}
We derive analogs of the Jarzynski equality and Crooks relation to characterize the nonequilibrium work associated with changes in the spring constant of an overdamped oscillator in a quadratically varying spatial temperature profile. The stationary state of such an oscillator is described by Tsallis statistics, and the work relations for certain processes may be expressed in terms of $q$-exponentials. We suggest that these identities might be a feature of nonequilibrium processes in circumstances where Tsallis distributions are found.
\end{abstract}

DOI: 10.1103/PhysRevE.92.022143

PACS number(s): 05.70.Ln

\section{INTRODUCTION}

Work relations are remarkable identities associated with the behavior of systems undergoing nonequilibrium thermodynamic processes. As the name suggests, they refer to the performance of mechanical work on a system brought about by a protocol of change in external force fields while the system exchanges heat with a coarsely specified environment. The key point is that they hold when the thermomechanical processing of a system takes place at an arbitrary rate, and not just in the quasistatic limit associated with equilibrium thermodynamics. They are therefore statements about real nonequilibrium dissipative behavior and have attracted attention since they provide a perspective on the nature of entropy production [1-12].

The identification of principles underlying the transfer of energy in the form of heat and work motivated the initial development of thermodynamics in the 19th century, but the construction of a framework to justify such principles from a microscopic perspective is still ongoing. Work relations, and the underlying fluctuation relations or theorems, rely on rather few assumptions about the dynamics of system components and have proved to be extremely valuable in this regard. One of the first results in this category was developed by Bochkov and Kuzovlev [13,14] to describe the statistics of nonconservative work performed on a system in an isothermal heat bath. The Evans-Searles fluctuation theorem $[15,16]$ provided insight into the behavior of deterministic complex systems driven by external mechanical and thermal interactions. A wider appreciation of fluctuation theorems, and of the significant development that was then underway in nonequilibrium statistical physics, followed from the derivation of the Jarzynski equality $[17,18]$, an elegant work relation that can be established from a number of perspectives. Further interest in the field was generated by the Crooks relation [19], a connection between the statistics of work performed in two isothermal processes that are driven in a mutually time-inverted fashion. Both relations require the system to start out in thermal equilibrium. Experimental relevance of these results has been demonstrated [20].

We wish to explore work relations that hold for a system exposed to an environment with a temperature gradient. The control, characterization, and exploitation of nonisothermal conditions at the nanoscale has received considerable attention in recent years [21-23]. Thermodynamic concepts such as system temperature can remain valid at these scales [24], though it is clear that temporal and spatial fluctuations will be important, aspects that can be accommodated through using stochastic thermodynamics as a theoretical description [10]. The basis of this approach is a framework of stochastic system dynamics in which the concept of a temperature gradient enters through a specification of the statistical properties of the environmental noise.

We have in mind applications to experimental systems such as tweezers [25] and nanomechanical devices [26] that can be used to manipulate small objects such as colloidal particles. There are challenges in establishing and controlling a temperature profile at submicron spatial scales, but progress has been made in this direction [27-29]. Models of heat transport can be used to describe the elevation of local temperature brought about by laser absorption in a liquid medium [30] and by Joule heating in conducting nanostructures [21], and it seems that a range of thermal profiles can be established using suitable arrangements of heat sources and sinks, together with choice of media [29,31].

In such a nonisothermal environment, a system cannot achieve thermal equilibrium, and our aim is to understand how the Jarzynski and Crooks results need to be adapted. Work relations have been considered for other systems that are prevented from reaching thermal equilibrium, such as glasses [32]. If the dynamics prevent or delay evolution towards the true equilibrium, a free energy of the final state might emerge as a valid concept, and so too might work relations.

We focus our attention on the specific case of a harmonically bound particle in a quadratically varying thermal profile. We employ overdamped stochastic dynamics to model the behavior of such a particle showing that is governed by Tsallis rather than Boltzmann statistics in the stationary state [33]. We find that by using a suitable notation, the two work relations can be written in a very intuitive form for certain classes of protocols. A similar generalization of the work relations to Tsallis statistics was proposed by Ponmurugan [34] in the context of a basis in Tsallis entropy. In contrast, our results emerge from a more standard thermodynamic framework where Gibbs-Shannon entropy represents the uncertainty of the state of a stochastic system. We review the nonisothermal stochastic dynamics of systems in Sec. II, describe the Tsallis oscillator in Sec. III, and then present analogs of the Jarzynski and Crooks results in Secs. IV and V. We consider whether existing experimental techniques could be used to establish nonisothermal conditions to a degree that suits our purpose in Sec. VI before giving our conclusions in Sec. VII. 


\section{DYNAMICAL FRAMEWORK}

Dynamical approaches that do not introduce noise are available [1,2], but a stochastic framework [35-37] has a number of intuitive and mathematical advantages [12]. We consider a system that is weakly coupled to a complex environment where the uncertainty in the interactions that take place between them as time passes is represented by a stochastic force in the system dynamics.

We focus attention on a particle that moves in one spatial dimension under the influence of a confining potential, a dissipative force proportional to the particle velocity and a stochastic force with a strength related to the local temperature of the environment but lacking autocorrelation. The dynamics in the overdamped limit are specified by

$$
\dot{x}=-\frac{1}{m \gamma} \frac{\partial \phi(x, t)}{\partial x}+\left(\frac{2 k_{B} T_{r}(x)}{m \gamma}\right)^{1 / 2} \xi(t),
$$

where $x$ is the particle position, $m$ is its mass, $\phi$ is the potential, $\gamma$ is the friction coefficient, $k_{B}$ is the Boltzmann constant, and $T_{r}$ is the temperature of the environment. The noise $\xi$ has statistical properties $\langle\xi(t)\rangle=0$ and $\left\langle\xi(t) \xi\left(t^{\prime}\right)\right\rangle=\delta\left(t-t^{\prime}\right)$, where the brackets denote an average over realizations. Equivalently we can express the dynamics using the following Itō-rules stochastic differential equation

$$
d x=-\frac{\phi^{\prime}}{m \gamma} d t+\left(\frac{2 k_{B} T_{r}(x)}{m \gamma}\right)^{1 / 2} d W,
$$

where $\phi^{\prime}=\partial \phi / \partial x$ and $d W$ is an increment in a Wiener process with $\langle d W\rangle=0$ and $\left\langle(d W)^{2}\right\rangle=d t$.

The work performed on the system as it follows a trajectory (denoted $\overrightarrow{\boldsymbol{x}}$ ) in an interval $0 \leqslant t \leqslant \tau$ is

$$
W[\overrightarrow{\boldsymbol{x}}]=\int_{0}^{\tau} \frac{\partial \phi[x(t), t]}{\partial t} d t=\int_{0}^{\tau} \dot{W}[x(t), t] d t,
$$

and for a constant environmental temperature $T_{r}=T_{0}$ it can readily be demonstrated $[12,17]$ that

$$
\left\langle\exp \left(-W / k_{B} T_{0}\right)\right\rangle=\exp \left(-\Delta F / k_{B} T_{0}\right),
$$

where $\Delta F=F\left(\phi^{\tau}\right)-F\left(\phi^{0}\right)$ is the difference in Helmholtz free energy, at temperature $T_{0}$, brought about by a change in the potential from $\phi^{0}=\phi(x, 0)$ to $\phi^{\tau}=\phi(x, \tau)$. The brackets denote an expectation over all trajectories possible under the dynamics, and represent either a path integration $\langle\cdot\rangle=$ $\int d \overrightarrow{\boldsymbol{x}} \mathcal{P}[\overrightarrow{\boldsymbol{x}}](\cdot)$ where $\mathcal{P}$ is a trajectory probability density, or the average $\langle\cdot\rangle=\int d W p(W)(\cdot)$ where $p$ is the probability density for the work done. Equation (4) is the celebrated Jarzynski equality, and it holds for an arbitrary work protocol as long as the system is initially in thermal equilibrium.

Under similar isothermal conditions and initial equilibrium, the Crooks relation

$$
p_{F}(W)=p_{R}(-W) \exp \left[(W-\Delta F) / k_{B} T_{0}\right]
$$

can be derived, where the suffices $F$ and $R$ refer to processes that are time-inverted partners, in that the potential evolves over time in process $R$ according to the reverse of the evolution in process $F$. The free energy difference in Eq. (5) refers to the outcome of the $F$ process.

Our purpose is to derive analogs of Eqs. (4) and (5) for work processes that take place in a nonisothermal environment in the limit of overdamped dynamics. The appropriate form of stochastic dynamics in these circumstances has recently received some attention [38,39], particularly with regard to thermodynamic differences arising from the neglect of housekeeping entropy production in an overdamped treatment [40]. The key point we exploit is that with overdamped dynamics the entropy production in the stationary nonisothermal state vanishes, such that it takes on something of the character of an equilibrium state, and this analogy allows us to construct an effective free energy change associated with a process and hence to derive work relations.

The approach we take is to recast the dynamics of Eq. (1) in an equivalent isothermal form

$$
\dot{y}=-\frac{1}{m \gamma} \frac{\partial \Phi(y, t)}{\partial y}+\left(\frac{2 k_{B} T_{0}}{m \gamma}\right)^{1 / 2} \xi(t),
$$

in terms of a stochastic variable $y(x)$ and an effective potential $\Phi(y, t)$, and to reinterpret the work relations that emerge in this representation. The mathematical reformulation is straightforward. Using Itō calculus we write

$d y=\left(-\frac{\phi^{\prime}}{m \gamma} \frac{d y}{d x}+\frac{k_{B} T_{r}}{m \gamma} \frac{d^{2} y}{d x^{2}}\right) d t+\frac{d y}{d x}\left(\frac{2 k_{B} T_{r}}{m \gamma}\right)^{1 / 2} d W$,

and we choose $y(x)$ such that $d y / d x=\left(T_{0} / T_{r}\right)^{1 / 2}$ and

$$
\frac{d^{2} y}{d x^{2}}=-\frac{T_{0}^{1 / 2} T_{r}^{\prime}}{2 T_{r}^{3 / 2}},
$$

where $T_{r}^{\prime}=d T_{r} / d x$, giving

$$
d y=\left(-\frac{\phi^{\prime}}{m \gamma}-\frac{k_{B} T_{r}^{\prime}}{2 m \gamma}\right)\left(\frac{T_{0}}{T_{r}}\right)^{1 / 2} d t+\left(\frac{2 k_{B} T_{0}}{m \gamma}\right)^{1 / 2} d W,
$$

so that the effective potential is

$$
\begin{aligned}
\Phi(y, t) & =\int_{0}^{y} d y\left(\phi^{\prime}+\frac{1}{2} k_{B} T_{r}^{\prime}\right)\left(\frac{T_{0}}{T_{r}}\right)^{1 / 2} \\
& =\int_{0}^{x(y)} d x\left(\phi^{\prime}+\frac{1}{2} k_{B} T_{r}^{\prime}\right)\left(\frac{T_{0}}{T_{r}}\right) .
\end{aligned}
$$

The effective isothermal work performed in this reformulation is

$$
W_{\mathrm{eff}}[\overrightarrow{\boldsymbol{y}}]=\int_{0}^{\tau} d t \frac{\partial \Phi[y(t), t]}{\partial t},
$$

while the effective free energy change is $\Delta F_{\text {eff }}=F\left(\Phi^{\tau}\right)-$ $F\left(\Phi^{0}\right)$, with $\Phi^{\tau}=\Phi(y, \tau)$ and $\Phi^{0}=\Phi(y, 0)$. The Jarzynski equality is

$$
\int d \overrightarrow{\boldsymbol{y}} \mathcal{P}[\overrightarrow{\boldsymbol{y}}] \exp \left(-W_{\mathrm{eff}}[\overrightarrow{\boldsymbol{y}}] / k_{B} T_{0}\right)=\exp \left(-\Delta F_{\mathrm{eff}} / k_{B} T_{0}\right),
$$

and all that remains is to cast the left-hand side as an expectation $\int d \overrightarrow{\boldsymbol{x}} \mathcal{P}[\overrightarrow{\boldsymbol{x}}] \exp (-\mathcal{F}[\dot{W}])$ in terms of a functional $\mathcal{F}$ of the rate of performance of work $\dot{W}(x, t)$.

\section{TSALLIS OSCILLATOR}

We illustrate how Eq. (12) can be interpreted for a model of one-dimensional (1D) particle motion in a harmonic confining potential and a temperature profile that varies quadratically 
with position measured from the center of the potential. We consider $\phi(x, t)=\phi_{\kappa}=\kappa(t) x^{2} / 2$ and

$$
T_{r}(x)=T_{0}\left(1+\frac{\kappa_{T} x^{2}}{2 k_{B} T_{0}}\right),
$$

where $\kappa_{T}$ is a parameter with the same dimensions as the spring constant $\kappa$. This system is of interest since the Fokker-Planck equation for the pdf $P(x, t)$ corresponding to the dynamics of Eq. (1) is

$$
\frac{\partial P}{\partial t}=\frac{\partial}{\partial x}\left(\frac{\kappa x}{m \gamma} P+\frac{k_{B}}{m \gamma} \frac{\partial\left(T_{r} P\right)}{\partial x}\right),
$$

for which the stationary solution is

$$
P_{\mathrm{st}}^{\kappa}(x)=\left[\frac{\kappa_{T}}{2 \pi k_{B} T_{0}}\right]^{1 / 2} \frac{\Gamma\left(1+\kappa / \kappa_{T}\right)}{\Gamma\left(\frac{1}{2}+\kappa / \kappa_{T}\right)}\left(1+\frac{\kappa_{T} x^{2}}{2 k_{B} T_{0}}\right)^{-\frac{\kappa}{\kappa_{T}}-1},
$$

namely a Tsallis distribution, proportional to a $q$-exponential function defined by

$$
e_{q}(z)=[1+(1-q) z]^{1 /(1-q)},
$$

here with $q=\left(\kappa+2 \kappa_{T}\right) /\left(\kappa+\kappa_{T}\right) \quad$ and $z=-(\kappa+$ $\left.\kappa_{T}\right) x^{2} / 2 k_{B} T_{0}$. Such distributions have received attention because of the ubiquity of experimental data that take this form [33]. They contrast with the more usual Gaussian characteristic of equilibrium situations, which correspond to the limit $q \rightarrow 1$, or $\kappa_{T} \rightarrow 0$ in this case. The work relations that we derive will refer to processes initiated when the system is in such a stationary state.

The effective potential $\Phi(y, t)=\Phi_{\kappa}(y)$ can be found in closed form for this model. We have

$$
\begin{aligned}
\Phi_{\kappa}(y) & =\int_{0}^{x(y)} d x\left(\kappa x+\frac{1}{2} \kappa_{T} x\right)\left(1+\frac{\kappa_{T} x^{2}}{2 k_{B} T_{0}}\right)^{-1} \\
& =\left(\kappa+\frac{1}{2} \kappa_{T}\right) \frac{k_{B} T_{0}}{\kappa_{T}} \ln \left(1+\frac{\kappa_{T} x^{2}}{2 k_{B} T_{0}}\right) .
\end{aligned}
$$

From this we can write

$$
W_{\text {eff }}=\int_{0}^{\tau} d t \dot{\kappa} \frac{k_{B} T_{0}}{\kappa_{T}} \ln \left(1+\frac{\kappa_{T} x^{2}}{2 k_{B} T_{0}}\right),
$$

so that the functional that appears in the Jarzynski equality is

$$
\mathcal{F}[\dot{W}]=\int_{0}^{\tau} d t \dot{W} \frac{2}{\kappa_{T} x^{2}} \ln \left(1+\frac{\kappa_{T} x^{2}}{2 k_{B} T_{0}}\right),
$$

using $\dot{W}=\dot{\kappa} x^{2} / 2$. This becomes clearer to interpret when written in the form

$$
\mathcal{F}[\dot{W}]=\int_{0}^{\tau} d t \frac{\dot{W}}{k_{B} T_{0}}\left[\frac{T_{0}}{\left(T_{r}-T_{0}\right)} \ln \left(\frac{T_{r}}{T_{0}}\right)\right] .
$$

The factor in square brackets is unity for an isothermal bath, which can be demonstrated by writing $T_{r}=T_{0}+\epsilon(x)$, such that for small $\epsilon$ we have $\left[T_{0} /\left(T_{r}-T_{0}\right)\right] \ln \left(T_{r} / T_{0}\right) \approx$ $\left(T_{0} / \epsilon\right)\left[\epsilon / T_{0}+O\left(\epsilon^{2}\right)\right]=1+O(\epsilon)$ after which we take $\epsilon \rightarrow$ 0 , and the factor is less than or equal to unity for nonisothermal conditions. For an isothermal bath we would write $\mathcal{F}=$ $W / k_{B} T_{0}$.

For a given $x(t)$, the functional $\mathcal{F}$ of the associated work rate can be determined and $\langle\exp (-\mathcal{F})\rangle$ set equal to $\exp \left\{-\left[F\left(\Phi_{\kappa(\tau)}\right)-F\left(\Phi_{\kappa(0)}\right)\right] / k_{B} T_{0}\right\}$ with

$$
\begin{aligned}
& \exp \left[-F\left(\Phi_{\kappa}\right) / k_{B} T_{0}\right] \propto \int d y \exp \left[-\Phi_{\kappa}(y) / k_{B} T_{0}\right] \\
& =\int d y\left\{\cosh \left[\left(\kappa_{T} / 2 k_{B} T_{0}\right)^{1 / 2} y\right]\right\}^{-2 \kappa / \kappa_{T}-1} \\
& =\pi^{1 / 2} \frac{\Gamma\left(\frac{1}{2}+\kappa / \kappa_{T}\right)}{\Gamma\left(1+\kappa / \kappa_{T}\right)}
\end{aligned}
$$

noting that $x=\left(2 k_{B} T_{0} / \kappa_{T}\right)^{1 / 2} \sinh \left[\left(\kappa_{T} / 2 k_{B} T_{0}\right)^{1 / 2} y\right] \quad$ so that $\Phi_{\kappa}(y) / k_{B} T_{0}=\left[2 \kappa / \kappa_{T}+1\right] \ln \cosh \left[\left(\kappa_{T} / 2 k_{B} T_{0}\right)^{1 / 2} y\right]$. In short, the Jarzynski equality for this system is

$$
\begin{gathered}
\left\langle\exp -\int_{0}^{\tau} d t \frac{\dot{W}}{k_{B} T_{0}}\left[\frac{T_{0}}{\left(T_{r}-T_{0}\right)} \ln \left(\frac{T_{r}}{T_{0}}\right)\right]\right\rangle \\
=\frac{\Gamma\left[\frac{1}{2}+\kappa(\tau) / \kappa_{T}\right]}{\Gamma\left[1+\kappa(\tau) / \kappa_{T}\right]} \frac{\Gamma\left[1+\kappa(0) / \kappa_{T}\right]}{\Gamma\left[\frac{1}{2}+\kappa(0) / \kappa_{T}\right]} .
\end{gathered}
$$

\section{JARZYNSKI EQUALITY FOR MULTISTEP PROCESSES}

We focus our attention on work processes consisting of a set of $N$ abrupt changes in the spring constant, i.e., shifts $\kappa_{i-1} \rightarrow \kappa_{i}$ at times $t=t_{i}$ for $i=1$ to $N$, with $\kappa_{0}=\kappa(0)$ and $\kappa_{N}=\kappa(\tau)$. The protocol of spring constant shifts is illustrated in Fig. 1 together with a representation of a stochastic trajectory $x(t)$ in the presence of a background temperature profile $T_{r}(x)$. Tsallis distributions $P_{\text {st }}^{\kappa}$ associated with stationary states at the beginning and end of the period are also shown: the system begins in a stationary state, but it need not end the process in such a condition. Note that a form of ergodic consistency holds here in the sense that the probability density is nowhere zero during the evolution: the stochastic dynamics can take the system from any initial position to any final position.

For such a multistep process the functional $\mathcal{F}$ becomes

$$
\mathcal{F}\left(\left\{\kappa_{i}\right\},\left\{x_{i}\right\}\right)=\sum_{i=1}^{N} \frac{\left(\kappa_{i}-\kappa_{i-1}\right)}{\kappa_{T}} \ln \left(1+\frac{\kappa_{T} x_{i}^{2}}{2 k_{B} T_{0}}\right),
$$

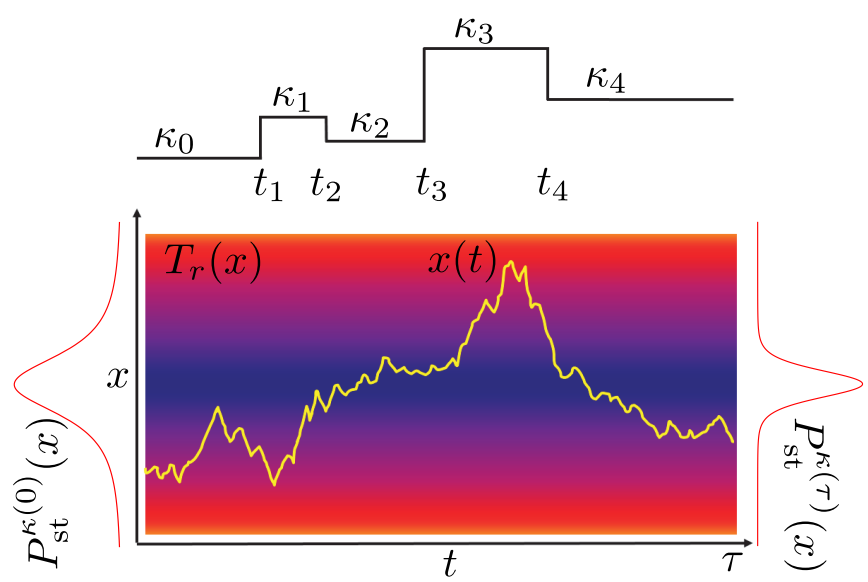

FIG. 1. (Color online) Illustration of a particle trajectory $x(t)$ in a nonisothermal environment (indicated by background color) as the spring constant changes in a sequence of steps. The initial distribution of positions takes the stationary Tsallis form $P_{\mathrm{st}}^{\kappa(0)}$. At time $\tau$ the distribution need not take the stationary form $P_{\mathrm{st}}^{\kappa(\tau)}$, shown for comparison. 
TABLE I. Demonstration of the Jarzynski equality for the Tsallis oscillator for a range of work processes consisting of a step up and down in spring constant (from $\kappa_{0}$ to $\kappa_{1}$ and back again) separated by a period $\tau=4$, with a variety of spatial temperature profiles specified by $\kappa_{T}$, in each case starting from a Tsallis distribution of position characterized by $q$, with $m=\gamma=k_{B}=T_{0}=\kappa_{0}=1$. Results are based on 1000 numerical realizations for each set of parameters, using a time step of $10^{-5}$. Errors in the mean are obtained assuming each realization is an independent sample. The left-hand side (LHS) of Eq. (25) is expected to equal unity.

\begin{tabular}{lllc}
\hline \hline$\kappa_{1} / \kappa_{0}$ & $\kappa_{T} / \kappa_{0}$ & $q$ & LHS of Eq. (25) \\
\hline 1.01 & 1.005 & 1.5 & $1.0000 \pm 0.0001$ \\
1.05 & 1.025 & 1.49 & $1.000 \pm 0.0011$ \\
1.10 & 1.05 & 1.49 & $1.000 \pm 0.0017$ \\
1.20 & 1.10 & 1.48 & $1.000 \pm 0.0021$ \\
1.40 & 1.20 & 1.46 & $1.001 \pm 0.0072$ \\
1.75 & 1.375 & 1.44 & $1.00 \pm 0.012$ \\
2.0 & 1.0 & 1.33 & $1.00 \pm 0.025$ \\
4.0 & 2.5 & 1.38 & $1.00 \pm 0.085$ \\
6.0 & 3.5 & 1.37 & $1.0 \pm 0.12$ \\
\hline \hline
\end{tabular}

where $x_{i}=x\left(t_{i}\right)$. Hence

$$
\begin{aligned}
\exp (-\mathcal{F}) & =\prod_{i=1}^{N}\left(1+\frac{\kappa_{T} x_{i}^{2}}{2 k_{B} T_{0}}\right)^{-\left(\kappa_{i}-\kappa_{i-1}\right) / \kappa_{T}} \\
& =\prod_{i=1}^{N} e_{q_{i}}\left(-\frac{\left(\kappa_{i}-\kappa_{i-1}\right) x_{i}^{2}}{2 k_{B} T_{0}}\right),
\end{aligned}
$$

where $q_{i}=1+\kappa_{T} /\left(\kappa_{i}-\kappa_{i-1}\right)$. This leads to the result

$$
\left\langle\prod_{i=1}^{N} e_{q_{i}}\left[-\frac{\Delta W_{i}}{k_{B} T_{0}}\right]\right\rangle=\frac{\Gamma\left(\frac{1}{2}+\kappa_{N} / \kappa_{T}\right)}{\Gamma\left(1+\kappa_{N} / \kappa_{T}\right)} \frac{\Gamma\left(1+\kappa_{0} / \kappa_{T}\right)}{\Gamma\left(\frac{1}{2}+\kappa_{0} / \kappa_{T}\right)},
$$

where $\Delta W_{i}=\left(\kappa_{i}-\kappa_{i-1}\right) x_{i}^{2} / 2$ is the work associated with the $i$ th step change in spring constant, such that the total work done is $W=\sum_{1}^{N} \Delta W_{i}$. The notation now makes very apparent the resemblance to the isothermal Jarzynski equality in Eq. (4). The brackets here refer to averaging according to $\langle\cdot\rangle=\int \prod_{0}^{N}(\cdot) d x_{i} p_{\mathrm{T}}\left(x_{0}, x_{1}, \ldots, x_{N}\right)$, where $x_{0}=x(0)$ and $p_{\mathrm{T}}$ is the probability that the dynamics generate a discrete trajectory $\left\{x_{i}\right\}$. If $\kappa_{T} \rightarrow 0$ the $q$-exponentials tend towards ordinary exponentials and the $\Gamma$ functions can be represented using Stirling's approximation such that the right-hand side of Eq. $(25)$ reduces to $\left(\kappa_{0} / \kappa_{N}\right)^{1 / 2}$, as expected.

It is straightforward to verify the identity (25) in simple cases such as a process consisting of a step up from $\kappa_{0}$ to $\kappa_{1}$ at time $t_{1}=0$ followed by a step back down to $\kappa_{2}=\kappa_{0}$ at $t_{2}=\tau$. We select initial positions according to the stationary distribution $P_{\mathrm{st}}^{\kappa_{0}}\left(x_{0}\right)$ given in Eq. (15) with a given $\kappa_{T}$, and solve the stochastic dynamics in Eq. (2) numerically in order to evaluate $x_{1}$ and $x_{2}$ and hence $\Delta W_{1}$ and $\Delta W_{2}$. The expectation of $e_{q_{1}}\left(-\Delta W_{1} / k_{B} T_{0}\right) e_{q_{2}}\left(-\Delta W_{2} / k_{B} T_{0}\right)$ over a number of realizations can be shown to be consistent with unity, within statistical uncertainty, as indicated in Table I. An illustration of the distribution of $\prod_{i=1}^{N} e_{q_{i}}\left(-\Delta W_{i} / k_{B} T_{0}\right)$ for a more complex cyclic process is shown in Fig. 2.

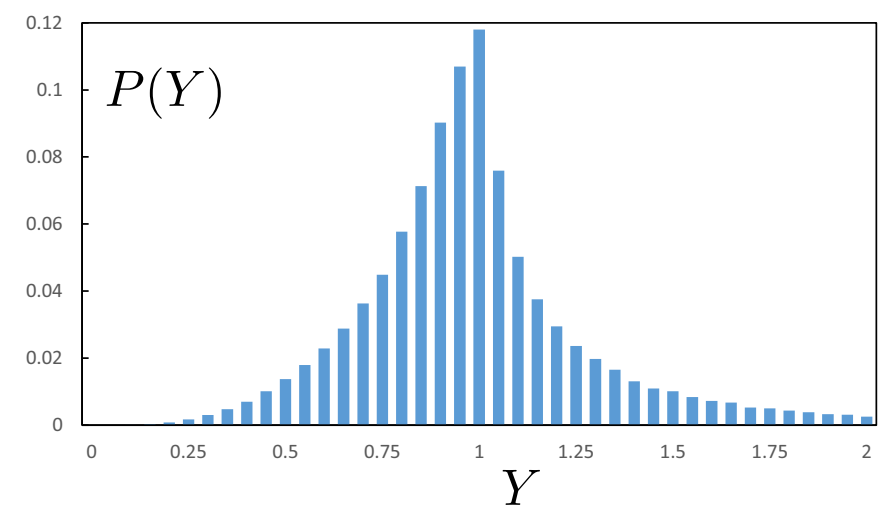

FIG. 2. (Color online) Distribution of values of $Y=$ $\prod_{i=1}^{4} e_{q_{i}}\left(-\Delta W_{i} / k_{B} T_{0}\right)$ for a cyclic process with $\kappa_{0}=1, \kappa_{1}=1.5$, $\kappa_{2}=2, \kappa_{3}=0.5$, and $\kappa_{4}=1$, with $t_{2}-t_{1}=t_{3}-t_{2}=t_{4}-t_{3}=0.1$, and $m=\gamma=k_{B}=T_{0}=\kappa_{T}=1$, obtained from $10^{5}$ realizations with a time step of $10^{-4}$. The mean of the distribution is 0.999 with statistical uncertainty of 0.001 , verifying the analog Jarzynski equality (25) for this process.

\section{CROOKS RELATION FOR A STEP PROCESS}

Our next task is to determine the analog of the Crooks relation for a Tsallis oscillator. The simplest demonstration involves an $F$ process consisting of a step up in spring constant from $\kappa_{0}$ to $\kappa_{1} \geqslant \kappa_{0}$ starting from a stationary state, such that the corresponding $R$ process is the step down from $\kappa_{1}$ to $\kappa_{0}$. It is straightforward to evaluate the statistics of work performed from the statistics of position prior to the step change. We write

$$
p_{F}(W)=P_{\mathrm{st}}^{\kappa_{0}}\left(x_{0}\right) \frac{d x_{0}}{d W},
$$

with $W=\left(\kappa_{1}-\kappa_{0}\right) x_{0}^{2} / 2 \geqslant 0$ such that $d x_{0} / d W=\left[2\left(\kappa_{1}-\right.\right.$ $\left.\left.\kappa_{0}\right) W\right]^{-1 / 2}$ and so with appropriate normalization we have

$$
\begin{aligned}
p_{F}(W)= & {\left[\frac{\kappa_{T}}{\pi k_{B} T_{0}} \frac{1}{\left(\kappa_{1}-\kappa_{0}\right) W}\right]^{1 / 2} \frac{\Gamma\left(1+\kappa_{0} / \kappa_{T}\right)}{\Gamma\left(\frac{1}{2}+\kappa_{0} / \kappa_{T}\right)} } \\
& \times e_{q_{0 F}}\left(-\frac{\left(\kappa_{0}+\kappa_{T}\right) W}{\left(\kappa_{1}-\kappa_{0}\right) k_{B} T_{0}}\right) \Theta(W),
\end{aligned}
$$

with $q_{0 F}=\left(\kappa_{0}+2 \kappa_{T}\right) /\left(\kappa_{0}+\kappa_{T}\right)$ and where $\Theta$ is the Heaviside function. Similarly we can determine the distribution of work $W=\left(\kappa_{0}-\kappa_{1}\right) x_{0}^{2} / 2 \leqslant 0$ for the reverse process starting from the stationary state with $\kappa=\kappa_{1}$ :

$$
\begin{aligned}
p_{R}(W)= & {\left[\frac{\kappa_{T}}{\pi k_{B} T_{0}} \frac{1}{\left(\kappa_{0}-\kappa_{1}\right) W}\right]^{1 / 2} \frac{\Gamma\left(1+\kappa_{1} / \kappa_{T}\right)}{\Gamma\left(\frac{1}{2}+\kappa_{1} / \kappa_{T}\right)} } \\
& \times e_{q_{0 R}}\left(-\frac{\left(\kappa_{1}+\kappa_{T}\right) W}{\left(\kappa_{0}-\kappa_{1}\right) k_{B} T_{0}}\right) \Theta(-W),
\end{aligned}
$$

with $q_{0 R}=\left(\kappa_{1}+2 \kappa_{T}\right) /\left(\kappa_{1}+\kappa_{T}\right)$. These two distributions are illustrated for the case of $\kappa_{0} / \kappa_{T}=1$ and $\kappa_{1} / \kappa_{T}=2$ in Fig. 3. 


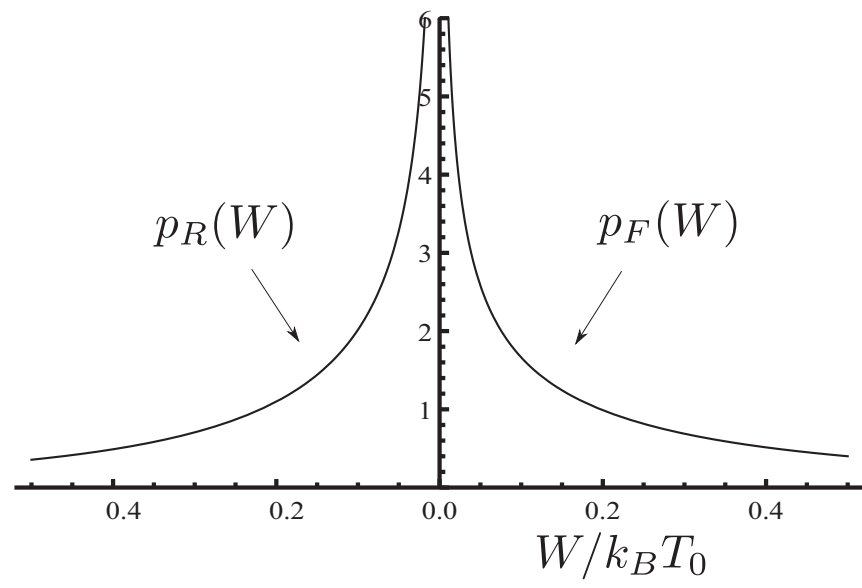

FIG. 3. Probability distributions of work for process $F$ consisting of a step up from $\kappa_{0}=\kappa_{T}$ to $\kappa_{1}=2 \kappa_{T}$ (positive values of $W$ ), and process $R$ specified by a step down from $\kappa_{1}$ to $\kappa_{0}$ (negative values of $W$ ), both starting from a stationary state. The mean work for the $F$ process is $k_{B} T_{0}$ and for the $R$ process it is $-k_{B} T_{0} / 3$.

The analog Crooks relation is then

$$
\begin{aligned}
\frac{p_{F}(W)}{p_{R}(-W)}= & \frac{\Gamma\left(\frac{1}{2}+\kappa_{1} / \kappa_{T}\right)}{\Gamma\left(1+\kappa_{1} / \kappa_{T}\right)} \frac{\Gamma\left(1+\kappa_{0} / \kappa_{T}\right)}{\Gamma\left(\frac{1}{2}+\kappa_{0} / \kappa_{T}\right)} \\
& \times\left(1+\frac{\kappa_{T} W}{\left(\kappa_{1}-\kappa_{0}\right) k_{B} T_{0}}\right)^{-\frac{\kappa_{0}}{\kappa_{T}}-1} \\
& \times\left(1-\frac{\kappa_{T} W}{\left(\kappa_{0}-\kappa_{1}\right) k_{B} T_{0}}\right)^{\frac{\kappa_{1}}{\kappa_{T}}+1},
\end{aligned}
$$

defined in this case for $W \geqslant 0$, which may be written in the form

$$
\begin{aligned}
\frac{p_{F}(W)}{p_{R}(-W)}= & \exp \left\{-\left[F\left(\Phi_{\kappa_{1}}\right)-F\left(\Phi_{\kappa_{0}}\right)\right] / k_{B} T_{0}\right\} \\
& \times\left(1+\frac{\kappa_{T} W}{\left(\kappa_{1}-\kappa_{0}\right) k_{B} T_{0}}\right)^{\left(\kappa_{1}-\kappa_{0}\right) / \kappa_{T}},
\end{aligned}
$$

and the final factor can be written as $e_{q_{R}}\left(W / k_{B} T_{0}\right)$ with $q_{R}=1-\kappa_{T} /\left(\kappa_{1}-\kappa_{0}\right)$ or $\left[e_{q_{F}}\left(-W / k_{B} T_{0}\right)\right]^{-1}$ with $q_{F}=1+$ $\kappa_{T} /\left(\kappa_{1}-\kappa_{0}\right)$. Once again, the notation makes it quite apparent that the Crooks relation for the pair of processes under isothermal conditions, in the form of Eq. (5), is recovered when $q_{F, R} \rightarrow 1$ and $\Phi_{\kappa} \rightarrow \phi_{\kappa}$ as $\kappa_{T} \rightarrow 0$.

We can further conclude from these distributions that the mean work performed in the $F$ process $\langle W\rangle_{F}=$ $\int W p_{F}(W) d W$ is given by $\left(\kappa_{1}-\kappa_{0}\right) k_{B} T_{0} /\left(2 \kappa_{0}-\kappa_{T}\right)$, as long as $\kappa_{0} \geqslant \kappa_{T} / 2$, and the mean work for the $R$ process is $-\left(\kappa_{1}-\kappa_{0}\right) k_{B} T_{0} /\left(2 \kappa_{1}-\kappa_{T}\right)$, assuming that $\kappa_{1} \geqslant \kappa_{T} / 2$. For a process consisting of a step up, the establishment of a stationary state, and a step down, the mean work would therefore be

$$
\langle W\rangle_{F}+\langle W\rangle_{R}=\frac{2\left(\kappa_{1}-\kappa_{0}\right)^{2} k_{B} T_{0}}{\left(2 \kappa_{0}-\kappa_{T}\right)\left(2 \kappa_{1}-\kappa_{T}\right)},
$$

which is never negative, and furthermore tends towards a known result [20] for such a process in the isothermal limit as $\kappa_{T} \rightarrow 0$.

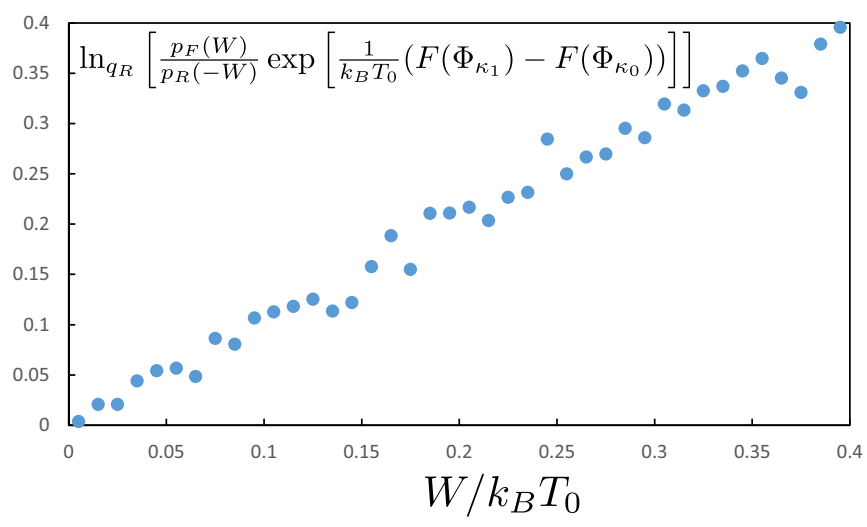

FIG. 4. (Color online) Verification of the analog Crooks relation Eq. (30) for a step up from $\kappa_{0}=\kappa_{T}$ to $\kappa_{1}=2 \kappa_{T}$ and a step down from $\kappa_{1}$ to $\kappa_{0}$, based on $10^{6}$ particle trajectories with a time step of $10^{-4}$, with $m=\gamma=k_{B}=T_{0}=\kappa_{T}=1$.

As an illustration of a possible experimental verification of the analog Crooks relation, we have generated trajectories according to Eq. (1) for a step up from $\kappa_{0} / \kappa_{T}=1$ to $\kappa_{1} / \kappa_{T}=2$, and the corresponding step down, both starting from the appropriate stationary state, in order to compute the work distributions. A plot of $\ln _{q_{R}}\left[p_{F}\left(W / k_{B} T_{0}\right) / p_{R}\left(-W / k_{B} T_{0}\right) \exp \left\{\left[F\left(\Phi_{\kappa_{1}}\right)-\right.\right.\right.$ $\left.F\left(\Phi_{\kappa_{0}}\right)\right] / k_{B} T_{0}$ \} against $W / k_{B} T_{0}$ should be a straight line with a gradient of unity, where $\ln _{q}(z)=\left(z^{1-q}-1\right) /(1-q)$ is the $q$-logarithm [33]. Such an outcome is apparent in Fig. 4.

\section{PRACTICAL CONSIDERATIONS}

The work relations will depart significantly from the form taken under isothermal conditions when $\kappa_{T} / \kappa \sim 1$ and we should consider whether current methods for establishing a thermal profile in a particle trap can provide such circumstances. Spring constants for particle trapping with optical tweezers in a liquid medium are typically of the order of $10^{-2}$ $\mathrm{pN} / \mathrm{nm}[28,41]$ and if we adopt this value for $\kappa_{T}$ in Eq. (13), the thermal gradient $\kappa_{T} x / k_{B}$ at a typical displacement of $10 \mathrm{~nm}$ from the trap center would be about $10 \mathrm{~K} / \mathrm{nm}$. This is not an unattainable gradient in a solid system $[21,26]$ but could pose experimental challenges for a fluid system.

In contrast, an approximately quadratic temperature profile over a spatial scale of microns with a peak thermal gradient of about $10 \mathrm{~K} / \mu \mathrm{m}$ can be established by laser illumination of a patterned nanostructure immersed in a fluid medium [29]. A particle can be confined within such a profile by thermophoresis (thermal diffusion) [42] or potentially by optical methods as well. Thermophoretic confinement provides much weaker trapping, with a spring constant of order $10^{-5} \mathrm{pN} / \mathrm{nm}$. The effect is equivalent to the presence of a potential given in dimensionless form by $\phi(x) / k_{B} T=S_{T} \Delta T_{r}(x)$, where $T$ is an effective constant ambient temperature and $S_{T}$ is the Soret coefficient that compares the strength of thermal diffusion, in response to a temperature gradient, with that of ordinary diffusion down a density gradient. For a quadratic temperature profile the confinement is harmonic with an effective spring constant $\kappa=T S_{T} \kappa_{T}$ and strongly nonisothermal conditions will therefore be established if $T S_{T} \sim 1$. Such a 
thermophoretic trap was demonstrated in [29] using $200 \mathrm{~nm}$ polystyrene spheres with $S_{T} \sim 3 \mathrm{~K}^{-1}$, and hence $\kappa_{T} \sim 10^{-3} \kappa$, but by trapping smaller species the Soret coefficient could potentially be reduced to $10^{-2} \mathrm{~K}^{-1}$. There remains the challenge of ensuring that such a trap is strong enough to retain a particle for a long enough time to perform measurements, and compromises will need to be made between the desired temperature profile and such requirements. Nevertheless, it seems possible that with further technological development the conditions of interest might be attainable.

\section{CONCLUSIONS}

Work relations exist for systems that are maintained away from thermal equilibrium by constraints, and a number of such cases have been discussed [43-45]. The situation we have explored here is of interest since the system in question, when described using overdamped dynamics, is governed by Tsallis statistics in its stationary state. Such statistical properties have been noted in a variety of physical systems [33], and contrast with the more usual Gaussian statistics of thermal equilibrium. The analogs of the Jarzynski equality and Crooks relation for such a system, constructed for certain processes, take a particularly transparent form when we make use of the $q$ exponential functions associated with Tsallis statistics.

Stochastic entropy production can provide the conceptual underpinning of work relations in nonisothermal as well as isothermal conditions, employing the usual GibbsShannon form rather than the Tsallis entropy. We believe that experimental verification of these identities might be possible in small systems where the appropriate nonisothermal environment can be established and controlled.

\section{ACKNOWLEDGMENTS}

We thank Janet Anders for her helpful support and comments on this work.
[1] G. Gallavotti and E. G. D. Cohen, Phys. Rev. Lett. 74, 2694 (1995).

[2] D. J. Evans and D. J. Searles, Adv. Phys. 51, 1529 (2002).

[3] A. Imparato and L. Peliti, Phys. Rev. E 72, 046114 (2005).

[4] G. E. Crooks and C. Jarzynski, Phys. Rev. E 75, 021116 (2007).

[5] C. Jarzynski, C. R. Phys. 8, 495 (2007).

[6] U. Seifert, Eur. Phys. J. B 64, 423 (2008).

[7] D. J. Evans and G. Morriss, Statistical Mechanics of Nonequilibrium Liquids, Second Edition (Cambridge University Press, Cambridge, 2008).

[8] S. Deffner and E. Lutz, Phys. Rev. E 77, 021128 (2008).

[9] M. Esposito, U. Harbola, and S. Mukamel, Rev. Mod. Phys. 81, 1665 (2009).

[10] U. Seifert, Rep. Prog. Phys. 75, 126001 (2012).

[11] I. J. Ford, Statistical Physics: An Entropic Approach (Wiley, Chichester, UK, 2013).

[12] R. E. Spinney and I.J. Ford, in Nonequilibrium Statistical Physics of Small Systems: Fluctuation Relations and Beyond, edited by R. J. Klages, W. Just, and C. Jarzynski (Wiley-VCH, Weinheim, 2013).

[13] G. Bochkov and Y. Kuzovlev, Physica A 106, 443 (1981).

[14] G. Bochkov and Y. Kuzovlev, Physica A 106, 480 (1981).

[15] D. J. Evans, E. G. D. Cohen, and G. P. Morriss, Phys. Rev. Lett. 71, 2401 (1993).

[16] D. J. Evans and D. J. Searles, Phys. Rev. E 50, 1645 (1994).

[17] C. Jarzynski, Phys. Rev. Lett. 78, 2690 (1997).

[18] C. Jarzynski, Phys. Rev. E 56, 5018 (1997).

[19] G. E. Crooks, Phys. Rev. E 60, 2721 (1999).

[20] J. C. Reid, D. M. Carberry, G. M. Wang, E. M. Sevick, D. J. Searles, and D. J. Evans, Phys. Rev. E 70, 016111 (2004).

[21] F. Menges, H. Riel, A. Stemmer, and B. Gotsmann, Nano. Lett. 12, 596 (2012).

[22] J. Millen, T. Deesuwan, P. Barker, and J. Anders, Nat. Nanotechnol. 9, 425 (2014).

[23] M. Mecklenburg, W. A. Hubbard, E. R. White, R. Dhall, S. B. Cronin, S. Aloni, and B. C. Regan, Science 347, 629 (2015).
[24] D. Reguera, J. M. Rubi, and J. M. G. Vilar, J. Phys. Chem. B 109, 21502 (2005).

[25] L. Neukirch and A. N. Vamivakas, Contemp. Phys. 56, 48 (2014)

[26] I. Santamaria-Holek, D. Reguera, and J. M. Rubi, J. Phys. Chem. C 117, 3109 (2013).

[27] H. Mao, J. R. Arias-Gonzaliez, S. B. Smith, I. Tinoco Jr., and C. Bustamante, Biophys. J. 89, 1308 (2005).

[28] P. Kumari, J. A. Dharmadhikari, A. K. Dharmadhikari, H. Basu, S. Sharma, and D. Mathur, Opt. Express 20, 4645 (2012).

[29] M. Braun and F. Cichos, ACS Nano 7, 11200 (2013).

[30] E. J. Peterman, F. Gittes, and C. F. Schmidt, Biophys. J. 84, 1308 (2003).

[31] M. Braun, A. Würger, and F. Cichos, Phys. Chem. Chem. Phys. 16, 15207 (2014).

[32] S. R. Williams, D. J. Searles, and D. J. Evans, J. Chem. Phys. 129, 134504 (2008).

[33] C. Tsallis, Introduction to Nonextensive Statistical Mechanics (Springer, Berlin, 2009).

[34] M. Ponmurugan, arXiv:1110.5153 [cond-mat.stat-mech].

[35] J. Kurchan, J. Phys. A: Math. Gen. 31, 3719 (1998).

[36] K. Sekimoto, Prog. Theor. Phys. Suppl. 130, 17 (1998).

[37] K. Sekimoto, Stochastic Energetics, Lecture Notes in Physics, Vol. 799 (Springer, Berlin, 2010).

[38] A. Celani, S. Bo, R. Eichhorn, and E. Aurell, Phys. Rev. Lett. 109, 260603 (2012).

[39] H. Ge, Phys. Rev. E 89, 022127 (2014).

[40] I. J. Ford, arXiv:1508.03488 [cond-mat.stat-mech].

[41] A. Rohrbach, Phys. Rev. Lett. 95, 168102 (2005).

[42] R. Piazza and A. Parola, J. Phys.: Condens. Matter 20, 153102 (2008).

[43] T. Hatano and S. I. Sasa, Phys. Rev. Lett. 86, 3463 (2001).

[44] T. Speck and U. Seifert, J. Phys. A: Math. Gen. 38, L581 (2005).

[45] R. E. Spinney and I.J. Ford, Phys. Rev. E 85, 051113 (2012). 